\title{
A Comparison of Implications in Orthomodular Quantum Logic-Morphological Analysis of Quantum Logic
}

\author{
Mitsuhiko Fujio \\ Department of Systems Design and Informatics, Faculty of Computer Science and Systems Engineering, \\ Kyushu Institute of Technology, Iizuka-shi 820-8502, Japan \\ Correspondence should be addressed to Mitsuhiko Fujio, fujio@ces.kyutech.ac.jp
}

Received 26 June 2012; Accepted 3 August 2012

Academic Editor: Shigeru Kanemitsu

Copyright (C) 2012 Mitsuhiko Fujio. This is an open access article distributed under the Creative Commons Attribution License, which permits unrestricted use, distribution, and reproduction in any medium, provided the original work is properly cited.

\begin{abstract}
Morphological operators are generalized to lattices as adjunction pairs (Serra, 1984; Ronse, 1990; Heijmans and Ronse, 1990; Heijmans, 1994). In particular, morphology for set lattices is applied to analyze logics through Kripke semantics (Bloch, 2002; Fujio and Bloch, 2004; Fujio, 2006). For example, a pair of morphological operators as an adjunction gives rise to a temporalization of normal modal logic (Fujio and Bloch, 2004; Fujio, 2006). Also, constructions of models for intuitionistic logic or linear logics can be described in terms of morphological interior and/or closure operators (Fujio and Bloch, 2004). This shows that morphological analysis can be applied to various non-classical logics. On the other hand, quantum logics are algebraically formalized as orhomodular or modular ortho-complemented lattices (Birkhoff and von Neumann, 1936; Maeda, 1980; Chiara and Giuntini, 2002), and shown to allow Kripke semantics (Chiara and Giuntini, 2002). This suggests the possibility of morphological analysis for quantum logics. In this article, to show an efficiency of morphological analysis for quantum logic, we consider the implication problem in quantum logics (Chiara and Giuntini, 2002). We will give a comparison of the 5 polynomial implication connectives available in quantum logics.
\end{abstract}

\section{Mathematical Morphology}

Mathematical morphology is a method of non-linear signal processing using simple settheoretic operations, which has the feasibility of extracting the characteristic properties of shapes $[1,2]$. In this paper we will adopt the formulation thereof generalized on lattices [3-7].

We identify a binary relation $R \subseteq X \times A$ and the correspondence from $X$ to $A$. Namely, $R(x)=\{a \in A \mid(x, a) \in R\}$ for $x \in X$. We call the relation $R$ with $X$ and $A$ exchanged, the transpose of $R$ and denote it by ${ }^{t} R$. 


\subsection{Dilation and Erosion}

Let $X, A$ be partially ordered sets. If for any family $\left\{x_{\lambda}\right\} \subseteq X$ of $X$ which has a supremum $\bigvee_{\lambda} x_{\curlywedge}$ in $X$, the image $\left\{f\left(x_{\curlywedge}\right)\right\} \subseteq A$ has the supremum $\bigvee_{\lambda} f\left(x_{\curlywedge}\right)$ in $A$ and $f\left(\bigvee_{\lambda} x_{\curlywedge}\right)=\bigvee_{\lambda} f\left(x_{\curlywedge}\right)$ holds, then we call the mapping $f: X \rightarrow A$ a dilation from $X$ to $A$. Similarly, by changing supremum by infimum, we may introduce an erosion. We call dilation and erosion morphological operations. For two elements $x \leq y$ of $X$, we have $x \vee y=y, x \wedge y=x$, the morphological operations are monotone.

Example 1.1 (morphology of set lattices [7]). Given sets $X$ and $A$, consider the lattices of their power sets $\mathcal{X}=2^{X}, \mathcal{A}=2^{A}$. Let $R$ be a binary relation in $X \times A$. Then the mappings $D_{R}: \mathcal{A} \rightarrow X$ and $E_{R}: \mathcal{A} \rightarrow \mathcal{X}$ defined by

$$
\begin{gathered}
D_{R}(B)=\{x \in X \mid R(x) \cap B \neq \emptyset\}, \\
E_{R}(B)=\{x \in X \mid R(x) \subseteq B\}
\end{gathered}
$$

are a dilation and an erosion, respectively.

From the transpose ${ }^{t} R$ we may similarly define the dilation and erosion $D_{t_{R}}: \mathcal{X} \rightarrow \mathcal{A}$, $E_{t_{R}}: x \rightarrow A$.

The importance of this example lies in the fact that all morphological operations between set lattices are expressed in this form, whence it follows that giving a framework of morphological operations and a binary relation $R$ are equivalent. In particular, in the Kripke semantics, accessibility relationships being binary operations between possible worlds, we contend that giving the Kripke framework amounts to giving morphological operations.

\subsection{Adjunctions}

Suppose two mappings $f: X \rightarrow A$ and $g: A \rightarrow X$ between partially ordered sets satisfy the condition

$$
f(x) \leq a \Longleftrightarrow x \leq g(a)
$$

for any $x \in X, a \in A$. Then the mapping pair $(f, g)$ is called an adjunction and is written as $f \dashv g$ and $f$ is called the lower adjoint of $g$, with $g$ is the upper adjoint of $f$. Notice that every adjoint is uniquely determined if exists.

Proposition 1.2. For two monotone mappings $f: X \rightarrow A$ and $g: A \rightarrow X$ between partially ordered sets to satisfy $f \dashv g$, it is necessary and sufficient that for any $x \in X, a \in A$, the relation

$$
f(g(a)) \leq a, \quad x \leq g(f(x))
$$

holds. following.

Relations between morphological operations and adjunctions are given by the 
Proposition 1.3. Let $X, A$ be partially ordered sets and $f: X \rightarrow A, g: A \rightarrow X$.

(1) If $f$ has the upper adjoint, then it is a dilation. Conversely, if $A$ is a complete upper semilattice, then a dilation has the upper adjoint.

(2) If $g$ has the lower adjoint, then it is an erosion. Conversely, if $X$ is a complete lower semilattice, then a dilation has the lower adjoint.

Example 1.4 (adjunctions of set lattices [7]). In Example 1.1, we have $D_{R} \dashv E_{t_{R}}$ and $D_{t_{R}} \dashv E_{R}$. Note that in each adjunction pair, dilation and erosion, $R$ and ${ }^{t} R$ are to be interchanged.

\subsection{Interior and Closure Operators}

An idempotent monotone mapping $f: X \rightarrow X$ on a partially ordered set $X$ is called a filter mapping. A filter mapping with extensibility $(x \leq f(x))$ is called a closure operator and one with antiextensibility $(f(x) \leq x)$ is called an interior operator.

Proposition 1.5. Let $X, A$ be partially ordered sets and $f: X \rightarrow A, g: A \rightarrow X$ and $f \dashv g$. Then $g \circ f: X \rightarrow X$ is a closure operator of $X$ and $f \circ g: A \rightarrow A$ is an interior operator of $A$.

Example 1.6 (closing and opening). The closure operator $E_{R} \circ D_{t_{R}}$ and the interior operator $D_{R} \circ E_{t_{R}}$ on $\mathcal{X}$ which are induced by the adjunctions in Example 1.4 are called closing and opening by $R$, respectively. Similarly, we may define the closing and opening by ${ }^{t} R$ as operators on $A$.

In any complete lattice, closure operators are characterized by the notion of Moore family [8], where a Moore family is a subset $M$ of a partially ordered set $X$ which satisfies the following condition. For any subset $S \subseteq M$, if $S$ has the infimum $\wedge S$ in $X$, then $\bigwedge S \in M$ holds true.

Proposition 1.7 (see $[7,8])$. Let X be a partially ordered set.

(1) For any closure operator $\varphi: X \rightarrow X$, the totality of all $\varphi$-closed sets $\mathfrak{F}_{\varphi}=\{x \in X \mid \varphi(x)=$ x) forms a Moore family.

(2) If $X$ is a complete lattice, then for any Moore family $M \subseteq X$, there exists a unique closure operator on $X$ such that $M=\mathfrak{F}_{\varphi}$ holds.

We may establish similar properties of interior operators by appealing to the duality of a Moore family [7].

\section{Quantum Logic}

We refer to $[9,10]$ for quantum logic and lattice theory associated to it and we assemble here the minimum requisites for the subsequent discussions.

For simplicity's sake, we assume that the lattice $L$ always has the maximum element 1 and the minimum element 0 throughout in what follows. 


\subsection{OL and OQL}

An ortho-complemented lattice $L$ is a lattice which has an involutive and complementary operation ${ }^{\prime}: L \rightarrow L$ reversing the order:

(1) $a \wedge a^{\prime}=0, a \vee a^{\prime}=1$.

(2) $\left(a^{\prime}\right)^{\prime}=a$.

(3) $a \leq b \Rightarrow b^{\prime} \leq a^{\prime}$.

If, moreover, for $a$ and its complement $a^{\prime}$, the modular relation

$$
b \leq a^{\prime} \Longrightarrow(a \vee b) \wedge a^{\prime}=b
$$

holds, then $L$ is called an orthomodular lattice.

An ortho-complemented lattice satisfying the modular relation

$$
c \leq b \Longrightarrow(c \vee a) \wedge b=c \vee(a \wedge b)
$$

for any $a, b$ is an orthomodular, but not conversely. A Boolean lattice is an orthocomplemented lattice satisfying the modular relation. The inclusion order among these classes of lattices is

Boolean $\subset$ modular ortho-complemented $\subset$ orthomodular $\subset$ ortho-complemented.

In general, we call collectively quantum logic $(Q L)$ both orthologic $(O L)$ modelled on an ortho-complemented lattice and orthomodular logic (OQL) modelled on an orthomodular lattice. An orthomodular lattice being an ortho-complemented lattice, we mostly work with OL, with additional mentioning of some special features intrinsic of OQL.

The language of QL consists of a countable number of propositional variables

$p, q, r, \ldots$, and logical connectives $\neg$ (negation), $\wedge$ (conjunction). Denote by $\alpha, \beta, \ldots$ the formulae with $\Phi$ their totality. The disjunction $\vee$ is defined as an abbreviation of $\neg(\neg \alpha \wedge \neg \beta)$.

\subsection{Kripke Semantics}

The pair $\mathfrak{F}=(\Omega, R)$ of the set of all possible worlds $\Omega \neq \emptyset$ and the reflexive and accessibility relations $R \subseteq \Omega \times \Omega$ is called a Kripke frame or orthogonal frame of OL. Intuitionally, the binary relation $\varpi \in R(\omega)$ means that $\omega$ and $\varpi$ are "not orthogonal". Indeed, defining $\omega \perp \varpi$ by $\varpi \notin$ $R(\omega)$, then we see that the reflexiveness corresponds to $\omega \not \perp \omega$, while symmetry to $\varpi \not L \omega \Rightarrow$ $\omega \not \varnothing \varpi$.

For any set of possible worlds $X \subseteq \Omega$, we define its ortho-complement set by

$$
X^{\prime}=\{\omega \in \Omega \mid \omega \perp \xi(\forall \xi \in X)\} .
$$

Then in view of this, the power set lattice $2^{\Omega}$ of $\Omega$ becomes ortho-complemented. The orthogonality of a set $X$ and the possible worlds $\omega$ is defined by

$$
\omega \perp X \Longleftrightarrow \omega \in X^{\prime} .
$$


Expressing the orthogonality in terms of morphological operations

$$
\omega \perp X \Longleftrightarrow \omega \in D_{R}(X)^{\complement}=E_{R}\left(X^{\complement}\right)
$$

In an orthogonal frame $\mathfrak{F}=(\Omega, R)$, we consider a special class of subsets called propositions in $\mathfrak{F}$, that is, $X$ is a proposition in $\mathfrak{F}$ means that $X^{\prime \prime}=X$ holds. As we shall see below, it immediately follows from the definition that formulae in OL may be interpreted by assigning propositions in an orthogonal frame.

Proposition 2.1. In an orthogonal frame $\mathfrak{F}=(\Omega, R)$, for $X \subseteq \Omega$ to be a proposition, it is necessary and sufficient that it is an $R$-closed set $\left(E_{R}\left(D_{R}(X)\right)=X\right)$ in the sense of morphology.

(Note that $R$ being symmetric, we have $D_{R}=D_{t_{R}}$.)

Proof. By (2.6), we have $X^{\prime}=D_{R}(X)^{\complement}$, whence

$$
X^{\prime \prime}=D_{R}\left(D_{R}(X)^{\complement}\right)^{\complement}=E_{R}\left(D_{R}(X)\right)
$$

Corollary 2.2. The totality $\mathfrak{P}_{\mathfrak{F}}$ of all propositions of $\mathfrak{F}$ forms a lower semi-complete orthocomplemented sublattice of $\mathbf{2}^{\Omega}$.

Proof. Note that $D_{R}(\emptyset)=\emptyset$, and that from reflexibility of $R$, we have $D_{R}(X)=X$, so that $\emptyset^{\prime \prime}=\emptyset, X^{\prime \prime}=X$. Hence, $\emptyset, X \in \mathfrak{P}_{\mathfrak{F}}$. Since $\mathfrak{P}_{\mathfrak{F}}$ is a Moore family, and a fortiori lower semi-complete. Also, since $D_{R} \circ E_{R} \circ D_{R}=D_{R}$, we have for $X \in \mathfrak{P}_{\mathfrak{F}},\left(X^{\prime}\right)^{\prime \prime}=\left(X^{\prime \prime}\right)^{\prime}=$ $\left(D_{R}\left(E_{R}\left(D_{R}(X)\right)\right)\right)^{\complement}=D_{R}(X)^{\complement}=X^{\prime}$, whence we obtain $X^{\prime} \in \mathfrak{P}_{\mathfrak{F}}$. Hence, $\mathfrak{P}_{\mathfrak{F}}$ is closed with respect to the complementation '.

Let $\Pi$ be a lower semi-complete ortho-complemented sub-lattice of $\mathfrak{P}_{\mathfrak{F}}$ and let $\rho: \Phi \rightarrow$ $\Pi$ be a mapping such that

(1) $\rho(\neg \alpha)=\rho(\alpha)^{\prime}$

(2) $\rho(\alpha \wedge \beta)=\rho(\alpha) \wedge \rho(\beta)$.

We call the set $\mathfrak{M}=(\Omega, R, \Pi, \rho)$ a Kripke model of OL, consisting of both this and the Kriplke frame $\mathfrak{F}=(\Omega, R)$.

If $\omega \in \rho(\alpha)$ holds true, we write $\omega \vDash_{\mathfrak{M}} \alpha$ and say that the formula $\alpha$ is true in the possible world $\alpha$. We call the formula $\alpha$ such that $\rho(\alpha)=\Omega$ is true in the model $\mathfrak{M}$ and write $\vDash_{\mathfrak{M}} \alpha$. More generally, if for any $\beta$ belonging to a set $T$ of formulae, we have $\rho(\beta) \subseteq \rho(\alpha)$, then we say that $\alpha$ is a consequence of $T$ in the model $\mathfrak{M}$ and write $T \vDash_{\mathfrak{M}} \alpha$. If further, these hold true in any models, then we say that they are logically true in or logical consequences of, OL respectively.

The Kripke semantics of the orthomodular logic OQL may be defined by considering as $\Pi$ only those satisfying the orthomodular condition

$$
X \nsubseteq Y \Longrightarrow X \cap(X \cap Y)^{\prime} \neq \emptyset .
$$




\section{Morphological Analysis of Implication Connectives}

\subsection{Implication Problem in $Q L$}

In quantum logic QL, the implication problem is important [10]. Not only those in quantum logic, but in general, an implication connective $\rightarrow$ is required to satisfy, for any model $\mathfrak{M}$, at least the conditions

(1) $\vDash_{\mathfrak{M}} \alpha \rightarrow \alpha$,

(2) If $\vDash_{\mathfrak{M}} \alpha$ and $\vDash_{\mathfrak{M}} \alpha \rightarrow \beta$, then $\vDash_{\mathfrak{M}} \beta$.

In QL, this condition may be stated as follows. For any Kripke model $\mathfrak{M}$, we have

$$
\vDash_{\mathfrak{M}} \alpha \longrightarrow \beta \Longleftrightarrow \rho(\alpha) \subseteq \rho(\beta) .
$$

Thus, we take (3.1) as a requirement for an implication connective $\rightarrow$ in QL [10]. Then we note that the forumula $\alpha \rightarrow \beta:=\neg \alpha \vee \beta$ in classical logic is not an implication connective in the sense of QL.

On the other hand, there are several candidates for implication connectives. However, there are only 5 polynomial ones in the sense that they are expressed in finitely many $\neg, \vee, \wedge$ [10]:

(i) $\alpha \underset{1}{\rightarrow} \beta:=\neg \alpha \vee(\alpha \wedge \beta)$,

(ii) $\alpha \underset{2}{\rightarrow} \beta:=\beta \vee(\neg \alpha \wedge \neg \beta)$,

(iii) $\alpha \underset{3}{\rightarrow} \beta:=(\neg \alpha \wedge \beta) \vee(\alpha \wedge \beta) \vee(\neg \alpha \wedge \neg \beta)$,

(iv) $\alpha \underset{4}{\rightarrow} \beta:=(\neg \alpha \wedge \beta) \vee(\alpha \wedge \beta) \vee((\neg \alpha \vee \beta) \wedge \neg \beta)$,

(v) $\alpha \underset{5}{\rightarrow} \beta:=(\neg \alpha \wedge \beta) \vee(\neg \alpha \wedge \neg \beta) \vee(\alpha \wedge(\neg \alpha \vee \beta))$.

These are the all candidates for polynomial implications in the free orthomodular lattice generated by two elements satisfying

$$
a \leq b \Longleftrightarrow a \underset{*}{\longrightarrow} b=1 \text {. }
$$

There is a distinction between OL and OQL in that they are really implications in the respective logic.

Theorem 3.1 (see [10]). The polynomial implications $\underset{i}{\rightarrow}(1 \leq i \leq 5)$ are all implications in $O Q L$ but none of them are so in $O L$.

Proof. Proof depends on the fact that for (3.1) to hold for each $i$, it is necessary and sufficient that $\varpi$ satisfies the orthomodular condition (2.8). For details we refer to [10].

Theorem 3.2. In $O Q L, \alpha \underset{i}{\rightarrow} \beta(i=1,2,4,5)$ are logical consequences of $\alpha \underset{3}{\rightarrow} \beta$, that is, in any Kripke model $\mathfrak{M}=(\Omega, R, \Pi, \rho)$, we have

$$
\rho(\alpha \underset{3}{\longrightarrow} \beta) \subseteq \rho(\alpha \underset{i}{\longrightarrow} \beta) \quad(i=1,2,4,5) .
$$


Proof. We fix a Kripke model $\mathfrak{M}=(\Omega, R, \Pi, \rho)$. Interpretation of each implication is as follows, where we denote the interpretations of the formulae $\alpha, \beta$ by $A=\rho(\alpha), B=\rho(\beta)$, respectively.

$\langle 1\rangle \rho(\alpha \underset{1}{\rightarrow} \beta)=\left(A \cap(A \cap B)^{\prime}\right)^{\prime}$,

$\langle 2\rangle \rho(\alpha \underset{2}{\rightarrow} \beta)=\left(B^{\prime} \cap\left(A^{\prime} \cap B^{\prime}\right)^{\prime}\right)^{\prime}$,

$\langle 3\rangle \rho(\alpha \underset{3}{\rightarrow} \beta)=\left(\left(A^{\prime} \cap B\right)^{\prime} \cap(A \cap B)^{\prime} \cap\left(A^{\prime} \cap B^{\prime}\right)^{\prime}\right)^{\prime}$,

$\langle 4\rangle \rho(\alpha \underset{4}{\rightarrow} \beta)=\left(\left(A^{\prime} \cap B\right)^{\prime} \cap(A \cap B)^{\prime} \cap\left(\left(A \cap B^{\prime}\right)^{\prime} \cap B^{\prime}\right)^{\prime}\right)^{\prime}$,

$\langle 5\rangle \rho(\alpha \underset{5}{\rightarrow} \beta)=\left(\left(A^{\prime} \cap B\right)^{\prime} \cap\left(A^{\prime} \cap B^{\prime}\right)^{\prime} \cap\left(A \cap\left(A \cap B^{\prime}\right)^{\prime}\right)^{\prime}\right)^{\prime}$.

Proof of $\rho(\alpha \underset{3}{\rightarrow} \beta) \subseteq \rho(\alpha \underset{1}{\rightarrow} \beta)$. It suffices to prove $A \subseteq\left(A^{\prime} \cap B\right)^{\prime} \cap\left(A^{\prime} \cap B^{\prime}\right)^{\prime}$, which reads in morphological operations,

$$
A \subseteq D_{R}\left(D_{R}(A)^{\complement} \cap B\right)^{\complement} \cap D_{R}\left(D_{R}(A)^{\complement} \cap D_{R}(B)^{\complement}\right)^{\complement}
$$

Taking complements of bothsides, we obtain

$$
\begin{aligned}
A^{\complement} & \supseteq D_{R}\left(D_{R}(A)^{\complement} \cap B\right) \cup D_{R}\left(D_{R}(A)^{\complement} \cap D_{R}(B)^{\complement}\right) \\
& =D_{R}\left(\left(D_{R}(A)^{\complement} \cap B\right) \cup\left(D_{R}(A)^{\complement} \cap D_{R}(B)^{\complement}\right)\right)
\end{aligned}
$$

by the definition of dilation. Then by adjunction, this is equivalent to the following:

$$
\begin{aligned}
E_{R}\left(A^{\complement}\right) & \supseteq\left(D_{R}(A)^{\complement} \cap B\right) \cup\left(D_{R}(A)^{\complement} \cap D_{R}(B)^{\complement}\right) \\
& =\left(E_{R}\left(A^{\complement}\right) \cap B\right) \cup\left(E_{R}\left(A^{\complement}\right) \cap D_{R}(B)^{\complement}\right) .
\end{aligned}
$$

The last equality follows from the duality between $D_{R}$ and $E_{R}$. However, this inclusion relation is always true.

Proof of $\rho(\alpha \underset{3}{\rightarrow} \beta) \subseteq \rho(\alpha \underset{2}{\rightarrow} \beta)$. It suffices to prove $B^{\prime} \subseteq\left(A^{\prime} \cap B\right)^{\prime} \cap(A \cap B)^{\prime}$, which can be done as in the proof of $\rho(\alpha \underset{3}{\rightarrow} \beta) \subseteq \rho(\alpha \underset{1}{\rightarrow} \beta)$.

Proof of $\rho(\alpha \underset{3}{\rightarrow} \beta) \subseteq \rho(\alpha \underset{4}{\rightarrow} \beta) .\left(A^{\prime} \cap B^{\prime}\right)^{\prime} \supseteq\left(\left(A \cap B^{\prime}\right)^{\prime} \cap B^{\prime}\right)^{\prime}$, or what amounts to the same thing, it is enough to show $A^{\prime} \cap B^{\prime} \subseteq\left(A \cap B^{\prime}\right)^{\prime} \cap B^{\prime}$. Further for this to be true, it is necessary and sufficient that $A^{\prime} \subseteq\left(A \cap B^{\prime}\right)^{\prime}$, which is true, being equivalent to $A \supseteq A \cap B^{\prime}$.

Proof of $\rho(\alpha \underset{3}{\rightarrow} \beta) \subseteq \rho(\alpha \underset{5}{\rightarrow} \beta)$. Enough to prove $(A \cap B)^{\prime} \supseteq\left(A \cap\left(A \cap B^{\prime}\right)^{\prime}\right)^{\prime}$ and the proof can be done in the same way as the proof of $\rho(\alpha \underset{3}{\rightarrow} \beta) \subseteq \rho(\alpha \underset{4}{\rightarrow} \beta)$.

\section{Conclusion}

By applying morphological analysis to a Kripke model in quantum logics, we have shown that $\overrightarrow{3}$ is the strongest among the 5 polynomial implication connectives in OQL. Once one 
sees the result, one may feel that one could do without morphological analysis. However, the point lies in whether by just looking at the defining equation $((\mathrm{i}) \sim(\mathrm{v}))$ or its interpretation, one could recognize the conclusion. Thus, the merit of morphological analysis seems to be its intuitive lucidness as "Calculus."

We would like to return to the analysis of connectives other than $\underset{3}{\rightarrow}$.

\section{References}

[1] G. Matheron, Random Sets and Integral Geometry, John Wiley \& Sons, 1975.

[2] J. Serra, Image Analysis and Mathematical Morphology, vol. 1, Academic Press, London, UK, 1984.

[3] J. Serra, Image Analysis and Mathematical Morphology, vol. 2, Academic Press, London, UK, 1988.

[4] C. Ronse, "Why mathematical morphology needs complete lattices," Signal Processing, vol. 21, no. 2, pp. 129-154, 1990.

[5] H. J. A. M. Heijmans and C. Ronse, "The algebraic basis of mathematical morphology. I. dilations and erosions," Computer Vision Graphics Image Processing, vol. 50, pp. 245-295, 1990.

[6] H. J. A. M. Heijmans, Morphological Image Operators, Academic Press, Boston, Mass, USA, 1994.

[7] M. Fujio and I. Bloch, "Non-classical logic via mathematical morphology," École Nationale Supérieure des Télécommunications, Technical Report ENST 2004D010, 2004.

[8] G. Birkhoff, Lattice theory, vol. 25 of American Mathematical Society Colloquium Publications, American Mathematical Society, Providence, RI, USA, 3rd edition, 1967.

[9] S. Maeda, Lattice Theory and Quantum Logic, Maki-Shoten, 1980.

[10] M. L. D. Chiara and R. Giuntini, "Quantum logics," in Handbooks of Philosophical Logic, D. M. Gabbay and F. Guenthner, Eds., vol. 6, pp. 129-228, Kluwer, Dordrecht, The Netherlands, 2nd edition, 2002. 


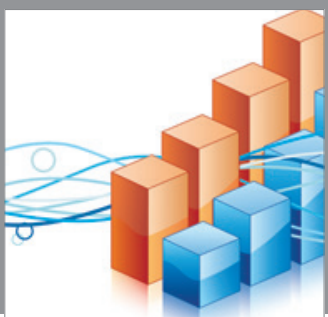

Advances in

Operations Research

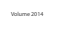

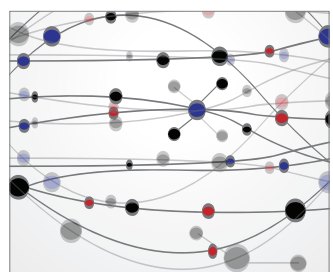

\section{The Scientific} World Journal
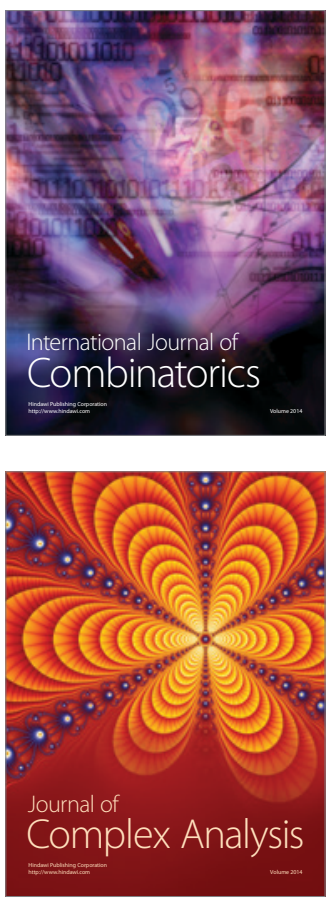

International Journal of

Mathematics and

Mathematical

Sciences
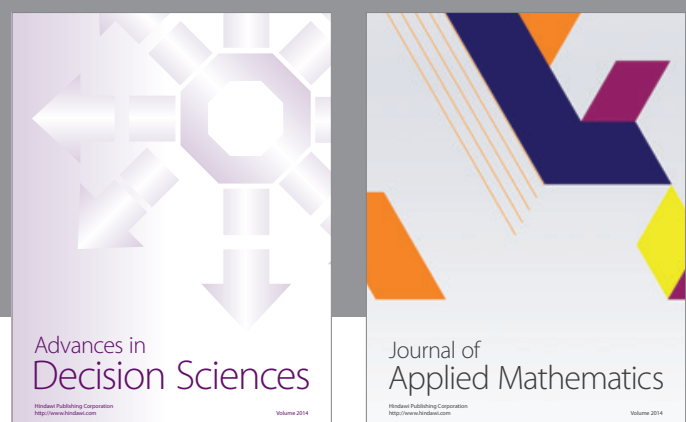

Journal of

Applied Mathematics
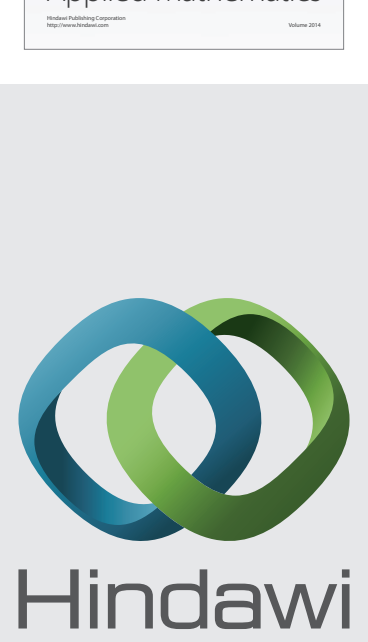

Submit your manuscripts at http://www.hindawi.com
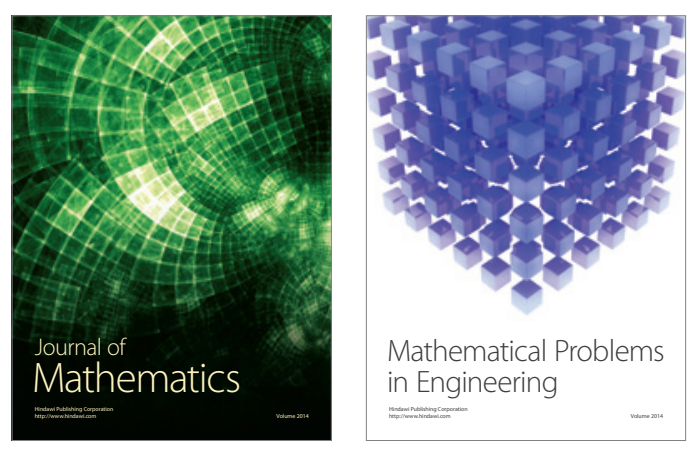

Mathematical Problems in Engineering
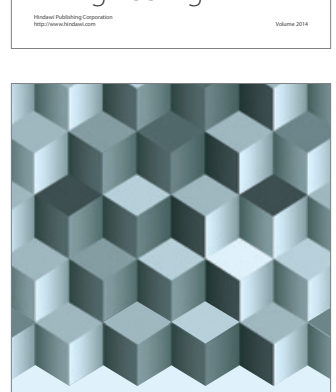

Journal of

Function Spaces
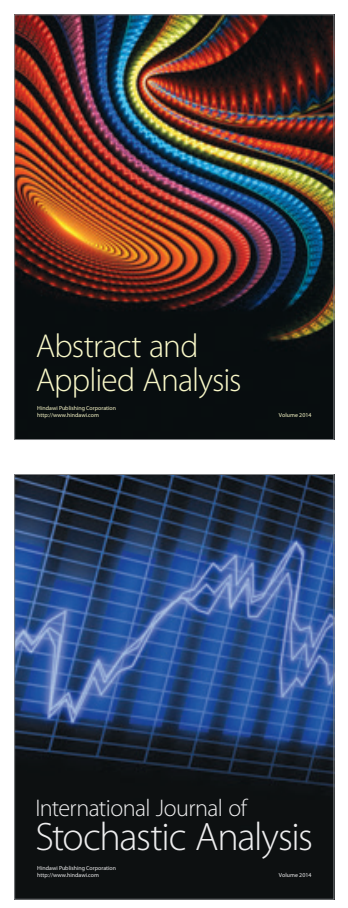

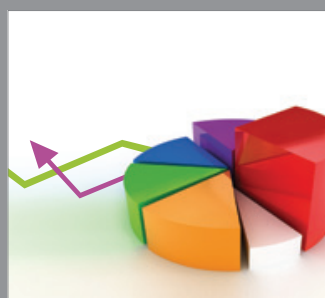

ournal of

Probability and Statistics

Promensencen
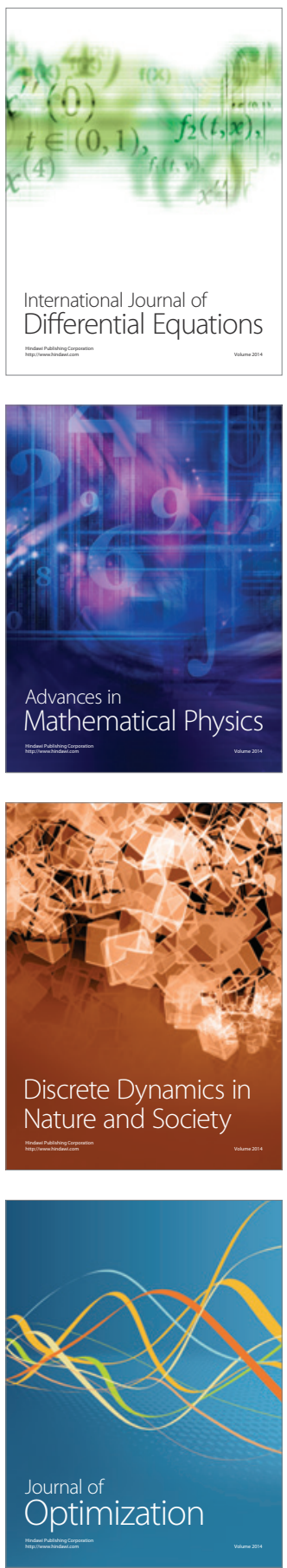